\title{
Trabeculectomia com mitomicina-C no tratamento de glaucomas refratários: avaliação dos resultados de 108
} casos

\author{
Mitomycin-C trabeculectomy in refractory glaucoma:evaluation of the results of 108 cases
}

Carmo Mandia Júnior ${ }^{1}$

Maria de Lourdes Veronese Rodrigues ${ }^{2}$
Universidade de São Paulo - Faculdade de Medicina de Ribeirão Preto.

Coleta de Dados: Departamento de Oftalmologia da Santa Casa de São Paulo.

${ }^{1}$ Primeiro Assistente Doutor do Departamento de Oftalmologia da Santa Casa de São Paulo; Professor Instrutor do Departamento de Oftalmologia da Faculdade de Medicina da Santa Casa de São Paulo.

${ }^{2}$ Livre-Docente do Departamento de Oftalmologia, Otorrinolaringologia e Cirurgia de Cabeça e Pescoço da Faculdade de Medicina da Universidade de São Paulo - USP - Ribeirão Preto.

Endereço para correspondência: Rua Madre Linda Lucotte, 174 - São Paulo (SP) CEP 05455-020.

E-mail: carmomandia@ig.com.br

Recebido para publicação em 04.05.2001

Aceito para publicação em 13.04.2002

Nota editorial: Pela análise deste trabalho e por sua anuência na divulgação desta nota, agradecemos aos Drs. Carlos Akira Omi e Paulo Augusto de Arruda Mello.

\begin{tabular}{|c|}
\hline RESUMO \\
\hline $\begin{array}{l}\text { Objetivo: Avaliar o comportamento da pressão intra-ocular (PIO) e a } \\
\text { possibilidade de eventuais complicações do uso da mitomicina-C (MMC) } \\
\text { nas trabeculectomias de pacientes com glaucoma refratário. Métodos: } \\
\text { Foram estudados prospectivamente } 108 \text { olhos de } 92 \text { pacientes submeti- } \\
\text { dos à trabeculectomia com mitomicina-C a } 0,2 \mathrm{mg} / \mathrm{ml} \text { por } 5 \text { minutos. O } \\
\text { tempo de seguimento foi de } 11,92 \pm 5,13 \text { meses, com mínimo de } 6 \text { e máximo } \\
\text { de } 24 \text { meses. A média de idade foi de } 39,59 \pm 24,05 \text { anos, variando de } 3 \\
\text { a } 87 \text { anos. A média da pressão intra-ocular no período pré-operatório dos } \\
108 \text { olhos foi de } 32,36 \pm 9,90 \text { mmHg, e a mediana de } 30 \text { mmHg, variando } \\
\text { de } 18 \text { a } 68 \text { mmHg. Resultados: A média da pressão intra-ocular no período } \\
\text { pós-operatório foi de } 13,74 \pm 9,68 \text { mmHg e a mediana de } 12 \text { mmHg, } \\
\text { variando de } 1 \text { a } 60 \text { mmHg. Houve sucesso em } 90,7 \% \text { dos olhos. Em } 76,8 \% \\
\text { não houve necessidade de medicação suplementar. Não houve diferença } \\
\text { significante (p > } 0,75) \text { quanto aos resultados pressóricos e às complica- } \\
\text { ções, nos pacientes submetidos ou não à intervenção prévia. Em } 25 \% \text { dos } \\
\text { olhos houve complicações; atalamia ( } 11,11 \% \text { e hipotonia ( } 7,41 \% \text { ) foram } \\
\text { as mais freqüentes. Conclusão: Trata-se de procedimento eficaz para o } \\
\text { tratamento de glaucomas refratários com baixo índice de complicações. }\end{array}$ \\
\hline
\end{tabular}

\section{INTRODUÇÃ̃O}

A trabeculectomia é o procedimento cirúrgico mais utilizado para tratar a grande maioria dos glaucomas ${ }^{(1)}$. $\mathrm{O}$ índice de sucesso da trabeculectomia nos casos de glaucoma primário de ângulo aberto varia de 80 a $90 \%{ }^{(2)}$. Entretanto, há um grupo de glaucomas, conhecidos como glaucomas refratários, que se caracterizam por grande resistência à redução da pressão intraocular (PIO), tanto com tratamento clínico tradicional como cirúrgico ${ }^{(3)}$. A abordagem cirúrgica dos glaucomas refratários inclui os procedimentos ciclodestrutivos, a instalação de dispositivos artificiais de drenagem, as cirurgias filtrantes tradicionais e a trabeculectomia ${ }^{(3)}$. O resultado cirúrgico da trabeculectomia nos glaucomas refratários varia de razoável a pobre ${ }^{(4)}$. Numerosos autores, analisando os diversos fatores que interferem no processo de cicatrização das cirurgias filtrantes, propõem o uso de diferentes drogas e métodos específicos com a finalidade de melhorar seus resulta$\operatorname{dos}^{(5-9)}$. Dentre as drogas antimetabólicas estudadas, a mitomicina-C (MMC) é a que maior interesse tem despertado, pelo fato de necessitar 
apenas uma única dose pré-operatória e pela ausência de toxicidade corneal ${ }^{(8)}$. Uma vez ativada, a MMC torna-se potente agente alquilante, inibindo a síntese de RNA, interrompendo a duplicação do DNA, a mitose e a síntese protéica ${ }^{(10)}$. Chen ${ }^{(11)}$ foi o primeiro a mostrar a eficácia da MMC nas cirurgias filtrantes. O objetivo deste estudo foi avaliar os efeitos da trabeculectomia associada à aplicação tópica pré-operatória de mitomicina-C, em olhos de pacientes portadores de glaucomas refratários, quanto à pressão intra-ocular e à possibilidade de eventuais complicações.

\section{MÉTODOS}

Foram estudados prospectivamente 108 olhos de 92 pacientes portadores de glaucomas refratários submetidos a trabeculectomia com aplicação tópica pré-operatória de mitomicina-C no período de novembro de 1991 e outubro de 1993, no Departamento de Oftalmologia da Santa Casa de São Paulo. Dos 92 pacientes, $49(53,3 \%)$ eram do sexo masculino e 43 $(46,7 \%)$ do sexo feminino. Cinqüenta e seis pacientes $(60,9 \%)$ eram da raça branca, $35(38,0 \%)$ da raça negra e $1(1,1 \%)$ da raça amarela. A idade dos pacientes variou de 3 a 87 anos, com média de 39,59 $\pm 24,05$. No nosso estudo, 60 olhos $(55,5 \%)$ foram submetidos a operações prévias, cuja quantidade variou de 1 a 5, com média de 1,55 $\pm 0,83$. Quarenta e oito olhos $(44,5 \%)$ não haviam sido submetidos a nenhuma operação prévia. O tempo mínimo de seguimento foi de 6 meses e o máximo de 24 meses. Os retornos dos pacientes no período pós-operatório foram programados para 1 dia, 1 semana, 1 mês, 3, 6, 9, 12, 15, 18, 21 e 24 meses. Os pacientes seguidos por apenas 6 meses foram examinados em 2 retornos, no mínimo, nesse período. A PIO pré-operatória variou de 18 a $68 \mathrm{mmHg}$, com média de 32,36 mmHg \pm 9,90 (Tabela 1 ).

\section{Técnica Cirúrgica}

Todas as cirurgias foram realizadas por 5 cirurgiões de acordo com a técnica descrita a seguir. Após a retopexia, realizava-se incisão da conjuntiva e da cápsula de Tenon, a aproximadamente $8 \mathrm{~mm}$ de distância do limbo. O quadrante escolhido para a intervenção cirúrgica ficava a critério do cirurgião. Depois da cauterização episcleral, um "cotonete" previamente embebido na solução de mitomicina $\mathrm{C}(0,2 \mathrm{mg} / \mathrm{ml})$ era colocado sobre a esclera e coberto pela cápsula de Tenon e conjuntiva. Após 5 minutos, o "cotonete" era retirado e realizava-se abundante lavagem do local, com $20 \mathrm{ml}$ de solução salina fisiológica. Em seguida, dissecava-se um retalho escleral superficial de forma quadrangular, com base límbica e medindo 4 por $4 \mathrm{~mm}$, até a córnea transparente. O retalho profundo de forma retangular, medindo $2 \mathrm{~mm}$ no sentido radial e $3 \mathrm{~mm}$ no sentido paralelo ao limbo, era removido e a iridectomia periférica realizada. $O$ retalho escleral superficial era suturado com dois pontos de mononylon 10,0, um em cada vértice. Sutura englobando cápsula de Tenon e conjuntiva era feita com pontos separados de mononylon 10-0. Injetava-se, no espaço subconjuntival do quadrante oposto ao da operação, solução contendo $0,5 \mathrm{ml}$ de gentamicina $(40 \mathrm{mg} / \mathrm{ml})$ e $0,5 \mathrm{ml}$ de dexametasona ( $4 \mathrm{mg} / \mathrm{ml})$. Finalmente, aplicava-se curativo oclusivo. No primeiro dia após a operação, o curativo oclusivo era retirado e iniciava-se a instilação de colírios contendo ciclopentolato, antibiótico e cortisona, por período mínimo de 15 dias ou até que os sinais inflamatórios intra-oculares tiveram desaparecido.

\section{Critérios de Inclusão}

Foram incluídos neste estudo olhos de indivíduos portadores de glaucomas com indicação cirúrgica e que apresentassem pelo menos uma das seguintes características:

1. Glaucoma refratário à trabeculectomia convencional, i.é, glaucoma secundário a uveítes, neovascular, glaucoma do afácico, glaucoma secundário a ceratoplastia penetrante, glaucoma traumático, glaucoma cortisônico ou pacientes submetidos previamente a uma trabeculectomia que falhou.

2. Glaucoma congênito primário em pacientes submetidos a múltiplos procedimentos, glaucoma juvenil ou qualquer paciente com idade inferior a 35 anos.

3. Glaucoma primário em pacientes da raça negra, no qual o olho contralateral apresentasse insucesso com cirurgia filtrante.

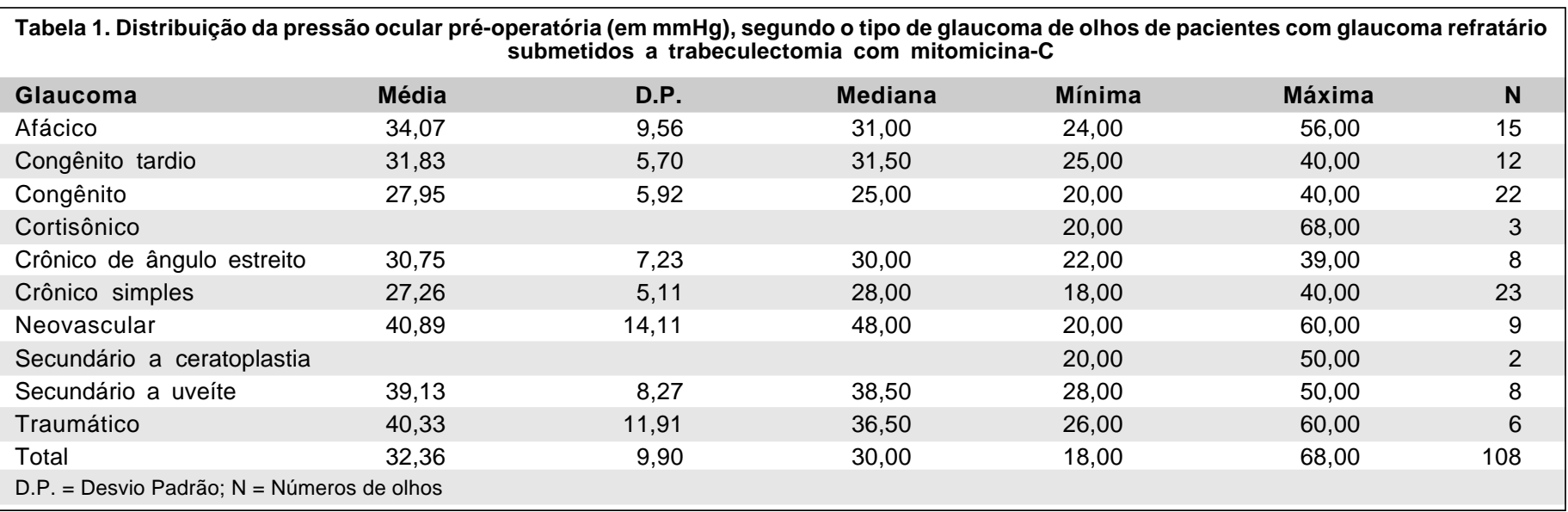




\section{Critérios de Sucesso}

1. Pressão intra-ocular final igual ou menor que $21 \mathrm{mmHg}$ e maior que $6 \mathrm{mmHg}$, com ou sem medicação, se a pressão intra-ocular inicial for maior ou igual a $26 \mathrm{mmHg}$.

2. Pressão intra-ocular final $25 \%$ menor que a pressão intraocular inicial e maior que $6 \mathrm{mmHg}$, com ou sem medicação, quando esta for menor que $26 \mathrm{mmHg}$.

Definimos como hipotonia a PIO menor que $6 \mathrm{mmHg}$, independente da presença de maculopatia hipotônica ou descolamento de coróide.

\section{Análise Estatística}

Para análise estatística dos resultados, empregou-se o teste exato de Fischer e o teste do qui-quadrado. Para análise da curva de probabilidade de sucesso, utilizou-se o método de Kaplan-Meier.

\section{RESULTADOS}

\section{Pressão intra-ocular}

A média final da PIO dos 108 olhos operados foi de 13, $74 \mathrm{mmHg} \pm 9,68$, variando de 1 a $60 \mathrm{mmHg}$ e mediana de $12 \mathrm{mmHg}$ (Tabela 2). O gráfico 1 mostra a curva de probabilidade de sucesso para a amostra total. Note-se que a probabilidade de sucesso diminui lentamente, atingindo $68 \%$ ao 18 meses de seguimento. A tabela 3 mostra os resultados obtidos nos diferentes tipos de glaucoma. Nos grupos congênitos tardio, cortisônico, crônico de ângulo estreito, secundário à ceratoplastia penetrante e secundário à uveíte, o índice de sucesso foi de $100 \%$. O grupo neovascular foi o que apresentou menor índice de sucesso $(55,56 \%)$. O teste exato de Fischer revelou nível descritivo de probabilidade $\mathrm{p}=0,097$, sugerindo que a diferença entre os grupos, com relação à proporção de sucesso, não é estatisticamente significante. A tabela 4 mostra que, segundo os critérios de sucesso 1 e 2, 98 olhos $(90,7 \%)$ obtiveram sucesso e 10 olhos $(9,3 \%)$ insucesso. Analisando os

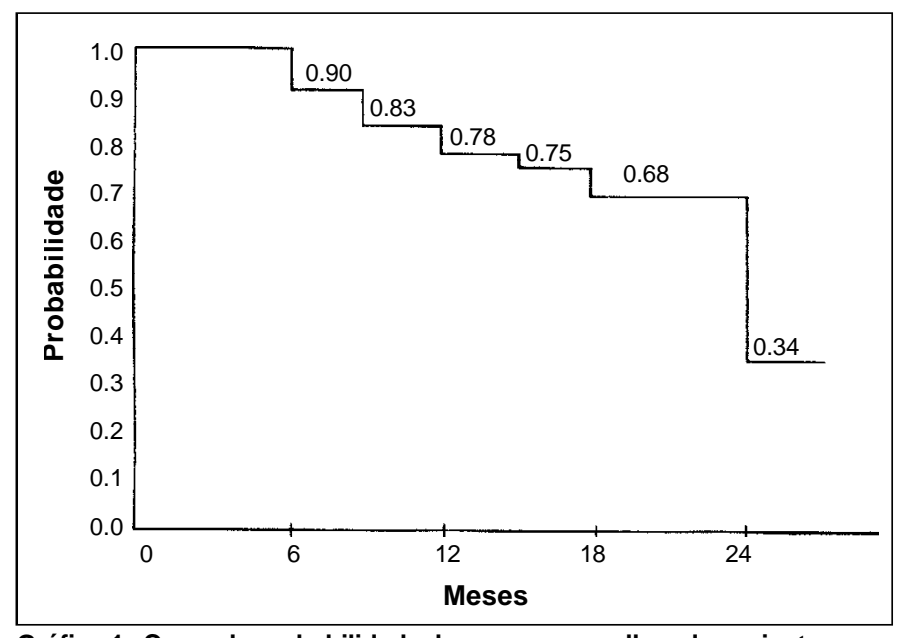

Gráfico 1 - Curva de probabilidade de sucesso em olhos de pacientes com glaucoma refratário submetidos a trabeculectomia com mitomicina-C

resultados segundo o critério 1 de sucesso, observamos que a média das pressões intra-oculares no período pré-operatório dos olhos que obtiveram sucesso era $34,8 \mathrm{mmHg}$, enquanto naqueles onde houve insucesso, de $49,1 \mathrm{mmHg}$.

Em relação ao sexo, não houve diferença significativa quanto aos resultados ( $\mathrm{p}>0,75)$. Dos 49 pacientes do sexo masculino, $44(89,8 \%)$ obtiveram sucesso e dos 43 pacientes do sexo feminino, $38(88,4 \%)$ obtiveram sucesso. Em relação à raça, não houve diferença, quanto aos resultados, entre as raças branca e negra $(\mathrm{p}>0,50)$. Dos 92 pacientes estudados, $o$ único da raça amarela obteve sucesso no controle da PIO. Dos 56 pacientes de raça branca, $51(91,0 \%)$ obtiveram sucesso, assim como $30(85,7 \%)$ dos 35 da raça negra. $O$ índice de sucesso, com ou sem medicação, foi de $90,7 \%$ (98 olhos). O percentual de olhos com sucesso sem medicação foi de 76,8\% (83 olhos). Não se observa diferença nos resultados quando se analisam os olhos que haviam ou não sido submetidos à intervenção cirúrgica prévia. Dos 48 olhos que não haviam sido submetidos à intervenção cirúrgica prévia, $43(89,6 \%)$ apresentaram sucesso e dos 60 olhos que haviam sido subme-

\begin{tabular}{|c|c|c|c|c|c|c|}
\hline Tipo de glaucoma & Média & D.P. & Mediana & Mínima & Máxima & $\mathbf{N}$ \\
\hline Afácico & 11,93 & 5,91 & 14,00 & 1,00 & 20,00 & 15 \\
\hline Congênito tardio & 9,17 & 3,59 & 8,50 & 4,00 & 14,00 & 12 \\
\hline Congênito & 10,14 & 3,75 & 10,00 & 2,00 & 18,00 & 22 \\
\hline Cortisônico & & & & 6,00 & 13,00 & 3 \\
\hline Crônico de ângulo estreito & 11,50 & 4,54 & 9,50 & 8,00 & 19,00 & 8 \\
\hline Crônico simples & 14,87 & 6,33 & 14,00 & 5,00 & 30,00 & 23 \\
\hline Neovascular & 29,22 & 23,03 & 16,00 & 6,00 & 60,00 & 9 \\
\hline Secundário a ceratoplastia & & & & 10,00 & 12,00 & 2 \\
\hline Secundário a uveíte & 14,88 & 3,04 & 15,50 & 8,00 & 18,00 & 8 \\
\hline Traumático & 17,83 & 12,16 & 15,50 & 5,00 & 40,00 & 6 \\
\hline Total & 13,74 & 9,68 & 12,00 & 1,00 & 60,00 & 108 \\
\hline
\end{tabular}




\begin{tabular}{|c|c|c|c|c|c|}
\hline \multirow{2}{*}{$\begin{array}{l}\text { Tipo de glaucoma } \\
\text { Afácico }\end{array}$} & \multicolumn{2}{|r|}{ Sucesso } & \multicolumn{2}{|c|}{ Insucesso } & \multirow{2}{*}{$\begin{array}{l}\text { Total } \\
15\end{array}$} \\
\hline & & $(93,33 \%)$ & & $(6,67 \%)$ & \\
\hline Congênito tardio & & $(100,00 \%)$ & 0 & $(0,00 \%)$ & 12 \\
\hline Congênito & & $(95,45 \%)$ & 1 & $(4,55 \%)$ & 22 \\
\hline Cortisônico & 3 & $(100,00 \%)$ & 0 & $(0,00 \%)$ & 3 \\
\hline Crônico de ângulo estreito & 8 & $(100,00 \%)$ & 0 & $(0,00 \%)$ & 8 \\
\hline Crônico simples & 20 & $(86,96 \%)$ & 3 & $(13,04 \%)$ & 23 \\
\hline Neovascular & 5 & $(55,56 \%)$ & 4 & $(44,44 \%)$ & 9 \\
\hline Secundário a ceratoplastia & 2 & $(100,00 \%)$ & 0 & $(0,00 \%)$ & 2 \\
\hline Secundário a uveíte & 8 & $(100,00 \%)$ & 0 & $(0,00 \%)$ & 8 \\
\hline Traumático & 5 & $(83,33 \%)$ & 1 & $(16,67 \%)$ & 6 \\
\hline Total & 98 & $(90,80 \%)$ & 10 & $(9,20 \%)$ & $108(100 \%)$ \\
\hline
\end{tabular}

tidos a uma ou mais operações prévias, 55 (91,6\%) obtiveram sucesso.

\section{Complicações}

O gráfico 2 mostra que atalamia $(11,11 \%)$, hipotonia $(7,41)$, descolamento de coróide (5,56\%) e edema macular transitório $(3,70 \%)$ foram as complicações mais freqüentes. Alguns olhos apresentaram mais de uma complicação.

\section{DISCUSSÃO}

Nossos resultados mostram que a diferença entre as médias das pressões intra-oculares pré-operatórias $(32,36 \pm 9,90 \mathrm{mmHg})$ e pós-operatórias $(13,74 \pm 9,68 \mathrm{mmHg})$ foi de $18,62 \pm 10,32 \mathrm{mmHg}$. Mermoud et al..$^{(12)}$, utilizando MMC nas trabeculectomias realizadas em glaucomas refratários de indivíduos da raça negra, encontram-se diferença entre as médias pré e pós-operatórias de 23,00 $\pm 12,6 \mathrm{mmHg}$. Considerando os critérios previamente estabelecidos, observamos que, entre os 108 olhos, houve sucesso em 98 (90,7\%). Em apenas 10 olhos (9,3\%) houve insucesso. Nossos resultados assemelharam-se aos encontrados na literatura. Palmer ${ }^{(13)}$ obteve $84 \%$ de sucesso; Kitazawa et al. ${ }^{(14)}$ obtiveram sucesso em $93,4 \%$ dos olhos. Os níveis de pressão ocular variam muito nos seis primeiros meses após qualquer cirurgia filtrante. Por isso, estudamos a curva de probabilidade de sucesso (método de Kaplan-Meyer) a partir do $6^{\circ}$ mês pós-operatório. Observamos uma queda lenta e gradativa na curva de maneira que, no $18^{\circ}$ mês pós-operatório, a probabilidade de sucesso foi de $68 \%$. Kupin et al. ${ }^{\left({ }^{15}\right)}$ obtiveram, na curva de probabilidade de sucesso, $91 \%$ aos 6 meses e $85 \%$ aos 18 meses, Mermoud et al. ${ }^{(12)}$ encontram 77,3\% de probabilidade de sucesso aos 18 meses do período pós-operatório. Quando analisamos os olhos que no período pré-operatório apresentavam PIO iguais ou maiores que $26 \mathrm{mmHg}$, observamos que, naqueles que obtiveram sucesso (PIO menores ou iguais a $21 \mathrm{mmHg}$ ), a média das PIO no período pré-operatório era $34,8 \mathrm{mmHg}$ e, naqueles que não obtiveram sucesso (PIO maiores que $21 \mathrm{mmHg}$ ), a média das PIO no período pré-operatório

\begin{tabular}{|c|c|c|c|}
\hline $\begin{array}{l}\text { Critério de } \\
\text { sucesso }\end{array}$ & Sucesso & Insucesso & Total \\
\hline 1 & $\begin{array}{l}71 \text { olhos } \\
(65,7 \%) \\
\text { Po pré } \\
X=34,8 \mathrm{mmHg}\end{array}$ & $\begin{array}{l}7 \text { olhos } \\
(6,5 \%) \\
\text { Po pré } \\
X=49,1 \mathrm{mmHg}\end{array}$ & 78 olhos \\
\hline 2 & $\begin{array}{l}27 \text { olhos } \\
(25,0 \%) \\
\text { Po pré } \\
X=22,7 \mathrm{mmHg}\end{array}$ & $\begin{array}{l}3 \text { olhos } \\
(2,8 \%) \\
\text { Po pré } \\
X=22,7 \mathrm{mmHg}\end{array}$ & 30 olhos \\
\hline Total & $\begin{array}{l}98 \text { olhos } \\
(90,7 \%)\end{array}$ & $\begin{array}{l}10 \text { olhos } \\
(9,3 \%)\end{array}$ & 108 olhos \\
\hline $\begin{array}{l}\text { Critério de suc } \\
26 \mathrm{mmHg} ; \mathrm{Cr} \\
\text { medicação, qu } \\
\text { no período pr }\end{array}$ & $\begin{array}{l}\text { Po final } \leq 21 \mathrm{mmHg} \\
\text { sucesso } 2 \text { = Po fin } \\
26 \mathrm{mmHg} \text {; PopréX } \\
\text { rio. }\end{array}$ & $\begin{array}{l}\text {, com ou sem medica } \\
\text { hal } 25 \% \text { < Po inicial, } \\
=\text { média das pressões }\end{array}$ & $\begin{array}{l}\text { ação, se Po } \geq \\
\text { com ou sem } \\
\text { intra-oculares }\end{array}$ \\
\hline
\end{tabular}

era bem mais elevada, isto é, de 49,1 mmHg. Esses dados sugerem que pressões intra-oculares pré-operatórias mais elevadas estão relacionadas a maiores índices de insucesso.

Não há relato na literatura sobre a influência do sexo nos resultados de operações antiglaucomatosas. Em relação à raça, sabemos que indivíduos da raça negra apresentam uma forma mais agressiva de glaucoma primário de ângulo aberto, o tratamento clínico é menos eficaz ${ }^{(16-17)}$. Além disso, o índice de sucesso das intervenções filtrantes em indivíduos da raça negra, portadores de glaucoma crônico simples, é muito baixa ${ }^{(17)}$ e pior ainda nos portadores de glaucoma refratário ${ }^{(18-19)}$. Nossos resultados não mostraram diferença estatisticamente diferente em relação ao abaixamento da PIO e freqüência de complicações quando comparados entre raça branca e negra e ao sexo.

Entre os 98 olhos que obtiveram sucesso, 83 deles (76,8\%) não necessitam de nenhuma medicação suplementar. Kitazawa et al. ${ }^{(14)}$ observaram que, entre os olhos que obtiveram sucesso, $83 \%$ estavam controlados sem medicação. Na literatura esse percentual variou de $62 \%$ (15) a $83 \%$ (12). 
Em relação ao número de operações prévias, não encontramos diferença nos resultados. Houve sucesso em $89,6 \%$ dos olhos que não haviam sido operados e em $91,6 \%$ nos que haviam sido submetidos a uma ou mais intervenções. Sabe-se que o sucesso da trabeculectomia depende da formação da bolsa filtrante e que para tanto é necessário que haja tecido conjuntival sadio, isto é, livre de aderências ou fibrose, muito comuns nos olhos já operados. Todos os 60 olhos de nossa amostra, que haviam sido submetidos a uma ou mais intervenções cirúrgicas prévias, apresentavam alguma área de conjuntiva sadia dentro dos $180^{\circ}$ superiores, permitindo a realização da trabeculectomia. Em nenhum caso a operação foi realizada dentro dos $180^{\circ}$ inferiores pois, nesses casos, o risco de infecção pós-operatória é maior ${ }^{(20)}$.

Atalamia foi a complicação mais freqüente. Nos 12 olhos $(11,11 \%)$ ela foi transitória, pois todos apresentaram melhora após tratamento clínico, que variou de 3 a 10 dias. Em 6 desses olhos $(5,56 \%)$, houve descolamento de coróide. Não houve nenhum caso de atalamia ou descolamento de coróide no pósoperatório tardio. Hipotonia ocular pós-operatória ocorre com freqüência nos olhos tratados com mitomicina ${ }^{(21)}$. No nosso estudo, encontramos hipotonia ocular (Po final $<6 \mathrm{mmHg}$ ) em 7 olhos $(6,48 \%)$, porém apenas 1 deles $(0,93 \%)$ desenvolveu maculopatia hipotônica. O único caso de endoftalmite $(0,93 \%)$ que encontramos no nosso estudo ocorreu num paciente de 29 anos, portador de glaucoma traumático, cuja PIO estava sob controle, no $21^{\circ}$ mês pós-operatório. Apesar do tratamento clínico, o olho evoluiu com pressões elevadas $(40 \mathrm{mmHg}) \mathrm{e}$ teve de ser reoperado. Descolamento de retina $(0,93 \%)$ ocorreu no olho de uma criança de 8 anos de idade, portadora de glaucoma do afácico. Essa complicação ocorreu no $15^{\circ}$ mês pós-operatório.

A mitomicina-C é utilizada atualmente, nos casos de glaucoma de difícil controle, como os glaucomas refratários deste estudo, em pacientes da raça negra, pelas razões já expostas. Recentes estudos têm demostrado os bons resultados em longo prazo, de trabeculectomias com mitomicina-C. Cheung et al. mostraram que a redução da PIO nas trabeculectomias com MMC se mantém por até 3 anos de seguimento ${ }^{(22)}$. A

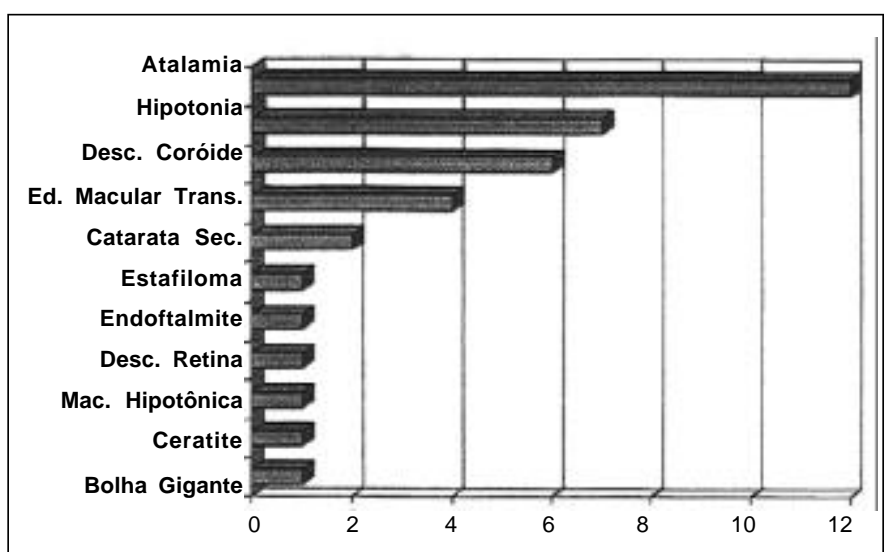

Gráfico 2 - Porcentagem de complicações em olhos de pacientes com glaucoma refratário submetidos a trabeculectomia com mitomicina-C
MMC tem se revelado útil no tratamento de outras formas de glaucoma. Sidoti et al. demonstraram a eficácia desta droga no tratamento de glaucomas pediátricos em que a goniotomia e/ ou trabeculotomia falharam. Entretanto, uma maior incidência de infecções relacionadas à bolha filtrante foi observada neste grupo $^{(23)}$. Lanzl et al. comprovaram a eficácia do uso da MMC nas trabeculectomias em pacientes com Síndrome Iridocorneo-endotelial ${ }^{(24)}$. Oito em 10 pacientes obtiveram controle da PIO durante o seguimento de 15 meses $^{(24)}$. Nosso estudo apresenta nossa experiência inicial com o uso de mitomicina-C nas trabeculectomias e vem corroborar a eficácia desta droga no tratamento coadjuvante dos glaucomas refratários.

\section{CONCLUSÃO}

A análise dos resultados de 108 trabeculectomias com mitomicina-C demonstrou que esta técnica é procedimento relativamente seguro e eficaz para o tratamento de glaucomas refratários.

\section{ABSTRACT}

Purpose: To evaluate the behavior of the intraocular pressure (IOP) and possible complications with the use of mitomycin-C (MMC) in trabeculectomies of patients with refractory glaucoma. Methods: 108 eyes of 92 patients who underwent mitomycin-C trabeculectomy $(0.2 \mathrm{mg} / \mathrm{ml}$ for 5 minutes) were studied. Mean follow up time was $11.92 \pm 5.13$ months (range, 6 to 24 months). Mean age was $39.59 \pm 24.05$ years (range, 3 to 87 years). Mean preoperative intraocular pressure was $32.36 \pm 9.90$ $\mathrm{mmHg}$, median $30 \mathrm{mmHg}$ (range, 18 to $68 \mathrm{mmHg}$ ). Results: Mean postoperative intraocular pressure was $13.74 \pm 9.68 \mathrm{mmHg}$, median $12 \mathrm{mmHg}$ (range, 1 to $60 \mathrm{mmHg}$ ). Success rate was $90.7 \%, 76.8 \%$ of patients needed no additional treatment. There was no statistical difference as to intraocular pressure and complication rate between primary and secondary cases. Twenty-five percent of the eyes presented complications; the most frequent were flat chamber $(11.11 \%)$ and hypotony (7.41\%). Conclusion: mitomycin-C trabeculectomy yields good results in refractory glaucoma, and has few complications.

Keywords: Trabeculectomy; Mitomycin/therapeutic use; Mitomycin/adverse effects; Open-angle glaucoma; Intraocular pressure/physiology

\section{REFERÊNCIAS}

1. Hoskins HD, Kass M. Surgery to relieve outflow block: external filtering procedures, In: Hoskins HD, Kass M. Becker-Shaffer's diagnosis and therapy of glaucomas. St. Louis,. Mosby; 1989. p.552-71.

2. Luntz HL. Surgical therapy of primary open angle glaucoma - filtering surgery for glaucoma. In: Cairins JE. Glaucoma. London: Grune e Stratton; 1986. p.593-632.

3. Almeida HG. Glaucoma neovascular In: Almeida HG, Almeida G. Glaucomas secundários. São Paulo: Roca; 1989. p.147-71. 
4. Katz LJ, Spaeth GL. Filtration surgery In: Ritch, Shields MB, Krupin T. The glaucomas. St Louis: Mosby; 1996. p.1989-98.

5. Skuta GL, Parrish II RK. Wound healing in glaucoma filtering surgery. Surv Ophthalmol 1987;32:149-70.

6. Alvarado J. The use of a liposome-encapsulated 5-fluoroorotate for glaucoma surgery: I. Animal studies. Trans Am Ophthalmol Soc 1989;87:489-514.

7. Herchler J. Long-term results of trabeculectomy with collagen sponge implant containing low-dose antimetabolite. Ophthalmology 1992;99:666-71.

8. Costa VP, Eiferman RA, Spaeth GL, Orengo-Nania S. Wound healíng modulation in glaucoma filtering surgery. Ophthalmic Surg 1993;24:152-70.

9. Lee DA, Goodwin T, Panek WC, Leong KW, Glasgow BJ. Effects of cytosine arabinoside-impregnated biorodible polymers on glaucoma filtration surgery in rabbits. J Glaucoma 1993;2:96-100.

10. Verweij J, Pinedo HM. Mitomycin-C: mechanism of action, usefulness and limitations. Anticancer Drugs 1990;1:5-13.

11. Chen CM. Enhanced intraocular pressure controlling effectiveness of trabeculectomy by local application of mitomycin-C. Trans Asia-Pacific Acad Ophthalmol 1983;9:172-7.

12. Mermoud A, Salmon JF, Murray AD. Trabeculectomy with mitomycin-C for refractory glaucoma in blacks. Am Jophthalmol 1993;116:72-8.

13. Palmer SS. Mitomycin as adjunct chemotherapy with trabeculectomy. Ophthalmology 1991;98:317-21.

14. Kitazawa Y, Kawase K, Matsushita H, Minobe M. Trabeculectomy with mitomycin: a comparative study with fluorouracil. Arch Ophthalmol 1991; 109:1693-8.
15. Kupin TH, Juzich MS, Shin DH, Khatana AK, Olivier MM. Adjunctive mitomycin $\mathrm{C}$ in primary trabeculectomy in phakic eyes. Am J Ophthalmol 1995;119:30-9.

16. Ramakrishnam R, Michon J, Robin AL, Krishnadas R. Safety and efficacy of mitomycin-C trabeculectomy in Southern India. A short-term pilot study. Ophthalmology 1993;100:1619-23.

17. Grehn F. World health problem of glaucoma. J Glaucoma 2001;10(5 Suppl.): S2-4.

18. Berson D, Zauberman H, Landau L, Blumenthal M. Filtering operations in Africans. Am J Ophthalmol 1969;67:395-8

19. Mermoud A, Salmon JF, Strakep C, Murray AND. The use of the single-plate Molteno implant in refractory glaucoma. Ophthalmologica 1992;205:113-20.

20. Wolner B, Liebmann JM, Sassani JW, Ritch R, Speaker M, Marmor M. Late bleb-related endophthalmitis after trabeculectomy with adjunctive 5-fluorouracil. Ophthalmology 1991;98:1053-60.

21. Shields MB, Scroggs MW, Sloop CM, Simmons RB. Clinical and histopathologic observations concerning hipotony after trabeculectomy with adjunctive mitomicyn-C. Am J Ophthalmol 1993;116:673-83.

22. Cheung JC, Wright MM, Murali S, Pederson JE. Intermediate-term outcome of variable dose mitomycin-c filtering surgery. Ophthalmology 1997;104:143-9.

23. Sidoti PA, Belmonte SJ, Liebmann JM, Ritch R. Trabeculectomy with mitomycin-c in the treatment of pediatric glaucomas. Ophthalmology 2000; 107:422-9.

24. Lanzl IM, Wilson RP, Dudley D, Augsburger JJ, Aslanides IM, Spaeth GL. Outcome of trabeculectomy with mitomycin-c in the iridocorneal endotelia syndrome. Ophthalmology 2000;107:295-7.

\section{Curso de Catarata $20 / 20$}

22 e 23 de Novembro de 2002

São Paulo - SP

INFORMAÇÕES: tel.: (11) 5575-4243 